\title{
Exploring hikikomori's information needs in Japanese context: applying a narrative inquiry approach with their dialogue
}

\author{
Hajime Naka \\ Tokoha University \& University of Tsukuba \\ h-naka@sz.tokoha-u.ac.jp
}

Keywords: hikikomori; social withdrawal; information needs; youth studies

In Japan, a growing number of youth is hiding from society and "confined to the inside [home]" (Ohashi, 2008). This syndrome of social withdrawal among youth is called "hikikomori", a phenomenological concept of social condition of remaining homebound for over 6 months avoiding any types of social participations. Hikikomori syndrome among Japanese youth was used to be seen as a psychiatric issue (Kato et al., 2012) but recently not only a psychiatric issue but also a socio-cultural issue (Saitō, 2013) due to their long-term condition of social isolation in Japanese society and culture.

One social-cultural issue that youth in hikikomori condition in Japan is that although many have needs to achieve social independence, these needs do not seem to motive their actual actions and behavior for going out from their house, which result in staying at home for long periods of time. This unfortunate disconnection between the need and the behavior among Japanese hikikomori youth poses the question of the nature of "information need" which is normally considered a cause of information seeking (Case \& Given, 2016) and thus a trigger and a driver of information seeking behavior (Savolainen, 2017) which, however, does not appear to apply to the case of Japanese youths in social withdrawal condition. In fact, in a comparative study on information needs of Finnish and 
Japanese hikikomoris conducted prior to this study, Japanese researcher found the difficulty in identifying information need from dialogues in a written format when using a simple content analysis approach due to hikikomoris' tendency of preferring to use an indirect and euphemistic expression of desire rather than more direct and concrete expressions of need for information seeking behaviors. Whereas information needs among Finnish youth can easily be identified from dialogues in one on one question format, it is very difficult to pinpoint an exact information need from Japanese youths dialogues in non-question, openquestion format.

For example, if a Japanese hikikomori say "I want money", what the meaning with this expression may vary depending on the context: sometimes a speaker simply needs money as a literal meaning, sometimes he/she needs social independence from parents, or sometimes he/she needs a methodology for a job search, or even his/her main purpose is to show some conundrums he/she is facing to somebody in order to receive social empathy from others instead of indicating a concrete information.

With this in mind, the researcher made a priori assumption that the way of expressing a "need" in Japanese culture can be very different from the one in other cultures, assuming that the expressions of "desire" in non-question format can be recognized as a possible information need in Japanese culture.

The purpose of this study thus is (1) to identify information need of hikikomori people from their dialogues in an online message board, and (2) to describe how and why their information needs impact negatively their motivation for staying at home by analyzing expressions of "desire" from their dialogues in mainly non-question format.

The researcher thus decided to apply a narrative inquiry approach and aimed to find their information needs not only from the contents but also within context of their dialogues. The narrative analysis is one of the analytical methods "for interpreting texts that have in common a storied form" (Riessman, 2008) in order to explore how and why incidents in speaker's lives are storied through which a researcher can understand the complexities of personal and social relations. Analytical methods in narrative analysis differ between academic discipline and analyst's perspectives. This study utilized Riessman's analytical methods. In doing so, the researcher intentionally selected 10 cases of written dialogue as a data set previously categorized as "economic problem" and "social relationship" from an online message board. Each dialogue was analyzed by taking both thematic and structural analysis approaches. In analyzing process the researcher focused on expressions about "need" and "desire" that can be considered as information need in their dialogues in an online message board called Hikkyin 2 ch, a SNS message boards posted by a user who is potentially or actually 
in hikikomori situation provided by $2 \mathrm{ch}$ site.

Three findings were revealed through the narrative analysis. Firstly, a thematic analysis revealed that several subjects emerged from each dialogue where an actor presented several interests and several information needs. When actor's interest changed, his/her information needs developed and even changed. For example, some hikikomori posted several messages starting for instance "I want to work" to "I asked my parents to give me a money" The following example shows the process of the transition of information needs in accordance with the level of priority.

"I want to go to school somewhere to get a license or a qualification. But I cannot imagine myself learning something at school. First of all, I don't have money, anyway."

Here, an information need for studying first occurred at a surface level. Then, it was negatively connected to a second information need, related to health/sickness or self-esteem. Finally, the person who posted the message arrives to the third information need, connected to an economic problem.

Secondly, a structural analysis revealed that actor's expressions about needs and desires has moved from an implicit/indirect level to specific/direct level or vice versa. In such transition, several actors' information needs developed and even changed from the one deeply embedded into context to a formalized one to even a compromised.

Thirdly, even the actor's interests seemed to be satisfied as their conversation flourished and even their information needs developed, their information need could not facilitate and trigger information behaviors to gain useful information in hikikomori context.

This study pioneers by providing an in-depth narrative analysis of the characteristic of information need of hikikomori, which also provide an explanation for why so many hikikomori are staying at home for long periods of time. The findings refine the feature of resigned, abandoned and compromised nature of information need throughout a sense-making process.

In future the information needs of Japanese and Finnish hikikomori are compared. The hypothesis is that cultural influence causes different kinds of information needs and expressions of information needs.

\section{References}

Case, D. O., \& Given, L. M. (2016). Looking for information: A survey of research on information seeking, needs, and behavior (Fourth edition). Bingley, UK: Emerald. 
Kato, T. A., Tateno, M., Shinfuku, N., Fujisawa, D., Teo, A. R., Sartorius, N., ... Kanba, S. (2012). Does the "hikikomori" syndrome of social withdrawal exist outside japan? A preliminary international investigation. Social Psychiatry and Psychiatric Epidemiology, 47(7), 1061-1075. https://doi.org/10.1007/s00127011-0411-7

Ohashi, N. (2008). Exploring the psychic roots of hikikomori in japan. UMI.

Riessman, C. K. (2008). Narrative methods for the human sciences. SAGE Publications.

Saitō, T. (2013). Hikikomori : Adolescence without end. University of Minnesota Press.

Savolainen, R. (2017). Information need as trigger and driver of information seeking: A conceptual analysis. Aslib Journal of Information Management, 69(1), 2-21. https://doi.org/10.1108/AJIM-08-2016-0139 